Debate sobre o artigo de Christopher Peterson Debate on the paper by Christopher Peterson

Donald Pollock

Department of Anthropology State University of New York at Buffalo Buffalo, USA.

\section{The social construction of medical metaphor}

Dr. Christopher Peterson's article on medical slang is an insightful analysis of the ways in which physicians and other medical staff use creative language to construct a response to contemporary crises in the health care system. I found little in Dr. Peterson's article with which to argue, and indeed, only wish that I had had access to his work when I addressed similar issues as they emerge in many of the autobiographical writings of physicians in the United States. My commentary is less a critique of his article than a very tentative consideration of some of the implications of his conclusions.

One of the first points that may strike the reader in the United States about the Brazilian medical slang that Dr. Peterson discusses is that it provides a fascinating perspective on institutions as much as on patients. As Dr. Peterson notes, much of the work done in the United States on medical slang, by David Gordon and others, focuses on the typification of patients through what is sometimes referred to as 'gomer' talk. This form of medical slang comprises a rich vocabulary of derogatory terms for patients rather than institutions. The symbolic core of this semantic field is the urban hospital emergency room, which indigent and poor patients use for their primary care, often presenting with minor medical problems, or with the complications of 'self-induced' conditions secondary to drug and alcohol use, cigarette smoking, and poor diet. Even 'good' patients may be spoken of in a mildly derogatory way, for example with the term 'YAVIS' or "young, attractive, verbal, intelligent, successful". The string of positive labels cannot quite hide the negative tone implied in the use of a stereotyping label.

Dr. Peterson's article makes two suggestions that could be turned back on medical slang in the United Stated very productively. First, he suggests that Brazilian medical slang encodes a relationship between health care providers and institutions, and indeed with the larger Brazilian political economy. Second, he suggests that the changes in health care that generate such perspectives are most productively considered to be Weberian rather than Marxian in nature.
Although Dr. Peterson is critical of Gordon and others for focusing exclusively on medical slang for patients and ignoring slang for institutions, I am not certain that, in the United States where Gordon did his research, physicians have developed a significant slang vocabulary for medical institutions. The one pertinent example that occurred to me while reading Dr. Peterson's article was the phrase 'doc in a box', referring to small clinics, often associated with larger hospitals or health maintenance organizations, that provide a wide range of primary care services to multiple zones within and around an urban area, like branches of a bank. The critical meanings embedded in the phrase allude to the (impression of) strippeddown, lower-quality of care provided by these facilities, with the image of a quick consultation from whichever physician happens to be staffing the 'box' at that time, combined with the notion of a cheap commodity sold in a generic box in large numbers. But apart from this example I am not familiar with any more complex slang vocabulary of the richness that Dr. Peterson uncovers in Rio de Janeiro, though this may represent my own myopia.

But Dr. Peterson's observation may also point to a certain social myopia in medical slang in the United States. One striking feature of 'gomer' talk is that it locates responsibility for a patient's conditions within the patient him - or herself, disregarding the larger social forces that lead one class of people to poverty and substance abuse, and another class of people to professional careers as physicians. I wondered, reading his article, if the relative lack of medical slang in the United States referring to physicians' relationship to hospitals and other institutions is a symptom of the radical individualism characteristic of US culture, where social forces are barely recognized, but individuals are presumed to have nearly complete control over all aspects of their lives and fates. This radical individualism emerged in interesting ways in the genre of physician autobiography I have called 'training tales', accounts of physicians' training in medical school or in residencies. The physician authors of these accounts describe their training experiences in the form of heroic quests in which they emerge as ennobled figures after undergoing a series of trials presented by difficult patients and dangerous hospitals, apparently mindless of the fact that the training institutions themselves are organized to produce the experiences and outcomes these physician-authors believe were unique to themselves. I also note that there are numerous slang expressions for other physi- 
cians, just as there are for patients, but hardly any at all for institutions.

I was therefore interested to see that Dr. Peterson focuses, correctly I think, on the bureaucratization of health care, and reads much of Carioca medical slang to be a response to this process. The alternative that has been explored extensively by physicians and medical social scientists of a more Marxist bent is the "proletarianization' of physicians, a process in which physicians increasingly find themselves serving as employees of de facto profit-making hospitals and 'managed care' corporations. In the mid-1980s journals such as The International Journal of Health Services Research were inflamed with angry analyses of Marxist scholars who read into this process the commodification of 'care' the conversion of a human relationship into a reified 'thing' that is produced, measured, withheld, or sold in a strictly economic model.

Dr. Peterson suggests, as I read him, that a more nuanced view of the recent history of the political economy of health care is necessary. After all, physicians have long worked as employees of institutions, and they are still far from being treated as hourly wage laborers. Rather, what has changed in the circumstances of physician work is the extraordinary level of bureaucratization it has undergone, at least in the United States and, apparently, in Brazil. I do not have the space in a brief commentary to develop this point in detail, but since it is not the explicit focus of Dr. Peterson's article I want to draw attention to the relevance of his argument for an ongoing debate in the sociology of medicine. While his Brazilian example has fascinating differences from the US cases with which I am most familiar, there are important similarities that make his article a significant contribution to this debate as well.

I should also note that this commentary is being written while I am traveling, and do not have access to the usual scholarly materials that I would want to consult; I apologize to Dr. Peterson and other authors if my memory fails me at one or two points in my discussion of their work.
James Trostle

Anthropology Program Trinity College Hartford, CT, USA.
Peterson presents compelling examples of the meaning and the constructions of medical slang in Rio de Janeiro, and describes the new meanings physicians create for (and communicate about) their health care system through their use of medical slang. But he makes unconvincing attempts to differentiate his study from others (particularly a study by Gordon in California), and sometimes moves too quickly between descriptions of metaphor, slang, jargon, proverbs, puns, and jokes. This is at once a pleasing and problematic paper, especially for a medical anthropologist with little formal sociolinguistic training.

Peterson contrasts substitutive, comparative, and interactive explanations of metaphor, and makes a convincing case for the utility of the third category. He presents only a sketch of the Brazilian health system crisis, but he makes appropriate and convincing references to a context of horror and moral challenge, and the development and use of medical slang to manifest and confront (or worsen) that context. On the other hand, his attempts to compare his study with one from California on hospital jokes (Gordon, 1983) are less convincing. He contrasts his broader with Gordon's narrower focus, though it is not clear that Gordon sought to represent anything other than one specific type of joke. He also critiques Gordon's attention to rapport among professionals and distance from patients, preferring instead his own attention to the creation of meaning. But while Peterson does attend to meaning, he also posits a mechanism of social critique that links a context of horror to the creation and use of puns by doctors. So is it that Peterson dislikes Gordon's lack of attention to meaning, or is it that Gordon employs a different functional model? Or both? Peterson later explains that he pays most attention to the third of his three themes (medical specialty, patients, health care services) because this is an area more relevant to the crisis. He chooses his own examples for what, as much as how, they communicate. Finally, Peterson writes that Gordon draws "curious conclusions." This critique seems to me to rest largely on a misrepresentation of the word 'claim,' as equivalent to the verb 'to demand' rather than the also acceptable 'to require' (contrary to Peterson's conclusion, in this latter sense comatose patients can readily claim attention).

But underneath this I am confused about the role of this critique in the article itself: without defending Gordon I wondered why Pe- 